\title{
La evaluación formativa y compartida como aspecto funda- mental en la formación del profesorado novel universitario. Experiencia en las Facultades de Ciencias y Farmacia de la Universidad de Granada
}

\author{
Isabel María Plaza del Pino*, Juan Manuel Bosque Sendra, Pilar Cerezo González, \\ Monsalud del Olmo Iruela y Cesar Viseras Iborra \\ Facultad de Ciencias y Facultad de Farmacia (Universidad de Granada)
}

(Received August 6, 2011; Accepted January 28, 2012)

\begin{abstract}
RESUMEN: Se presenta la experiencia de un equipo docente de la Universidad de Granada, constituido por cinco profesores experimentados y trece principiantes pertenecientes a las áreas de ciencias experimentales y de la salud. En dicha experiencia se han llevado a cabo una serie de acciones de formación, reflexión y puesta en práctica de estrategias dirigidas a la mejora del proceso de enseñanzaaprendizaje.
\end{abstract}

En este artículo se detallan: los antecedentes del equipo, sus intereses e inquietudes y la situación actual especificando las actividades realizadas y los resultados obtenidos en el trabajo desarrollado con el profesorado principiante.

Entre las tareas desarrolladas podemos destacar las innovaciones realizadas en evaluación formativa y compartida en asignaturas de características muy diversas, pertenecientes a las titulaciones de Bellas Artes, Biología, Ciencias Ambientales, Farmacia, Ingeniería Química y Química. Asimismo la experiencia del equipo como grupo de trabajo cooperativo ha servido de punto de referencia tanto a los profesores noveles como a los experimentados.

Palabras clave: formación de profesorado universitario, mentorización, equipo docente, evaluación para el aprendizaje, innovación docente.

Formative and shared assessment as a key aspect in the training of novice university teacher. Field experience in the Faculties of Science and Pharmacy at the University of Granada.

\footnotetext{
ABSTRACT: We present the experience of a teaching team at the University of Granada made up of five experienced teachers and thirteen novices all working in the areas of Health Sciences and Experimental Sciences. This experience involved processes of training, self-criticism and putting into practice some strategies aimed at improving the teaching-learning process.

In this article the following points are covered: The background of the team and the current situation, specifically the activities carried out and the results obtained with respect to the novice teachers. Among the developments we can point out innovations in Formative and Shared Evaluation in diverse subjects within the departments of Fine Arts, Biology, Environmental Science, Pharmacy, 
Chemical Engineering, and Chemistry. The experience of the teaching team as a collaborative workgroup is useful not only to the novice teachers but also to the experienced teachers.

Keywords: higher education teacher training, mentoring, teaching team, assessment for learning, teaching innovation.

\section{INTRODUCCIÓN}

Dentro del actual panorama de convergencia europea se nos propone un cambio que afecta al estilo docente del profesorado universitario. Es evidente que si se pretende avanzar hacia un modelo centrado en el aprendizaje es imprescindible que se produzca una evolución en la mentalidad del docente (Madrid, 2005). En este sentido, la formación del profesorado novel adquiere un papel relevante. Para abordar esta situación en los últimos años se están llevando a cabo, en diversas universidades españolas, acciones específicas de mentorización (Sánchez, 2008).

El profesor mentor podría entenderse como un experto con capacidades profesionales adquiridas por la experiencia y por años de formación continuada (Escudero, 2009). Su objetivo principal es ayudar a la integración de los miembros más jóvenes en todos los aspectos de la institución, y especialmente en el desempeño de la docencia. Como aspecto fundamental debe transmitir que la reflexión/revisión sobre la propia labor docente constituye una actividad de vital importancia como medio de mejora continua.

Paralelamente esta metodología favorece la creación de equipos docentes (Mayor y Sánchez, 1999) en los que participan conjuntamente profesorado principiante y experimentado que interactúan como fuente de cambio en el ámbito educativo universitario. De esta forma, además de promover la formación del nuevo profesorado y su integración en la vida universitaria, se facilita la reflexión del profesorado experimentado sobre su propia labor docente.

En los últimos años el Vicerrectorado para la Garantía de la Calidad de la Universidad de Granada está promoviendo la constitución de estos equipos docentes (Convocatoria de Apoyo a la Formación del Profesorado Principiante y Mejora de la Docencia 2009, 2010 y 2011). Los autores de este trabajo somos responsables de un programa formativo dentro de la convocatoria 2010, que acaba de ser renovado en la convocatoria 2011. En este artículo se presenta nuestra experiencia en el equipo docente y se detalla cómo se está desarrollando uno de los ejes principales de nuestro programa: la formación de los participantes en evaluación formativa y compartida.

Avanzar hacia una actividad docente centrada en el aprendizaje del alumnado afecta a todos los elementos curriculares y en especial a la evaluación que debe evolucionar para ajustarse a esta nueva situación. La evaluación condiciona poderosamente los procesos de aprendizaje y, por tanto, ninguna innovación curricular será efectiva si no va acompañada de innovaciones en el modo de concebirla. La forma más adecuada de abordar esta tarea implica transitar de una 
evaluación del aprendizaje a una evaluación para el aprendizaje (López, 2009; Sanmartí, 2007). Numerosos autores destacan el papel determinante que tiene la evaluación en cómo, cuándo y qué aprenden nuestros estudiantes (Brown y Glasner, 2003; Dochy, Segers y Dierick, 2002; López, 2009; Sanmartí, 2007). Estos autores también analizan cómo la autoevaluación, la evaluación entre compañeros y la coevaluación promueven la autorreflexión y el control sobre el propio aprendizaje. Por tanto, si somos capaces de diseñar los programas de nuestras asignaturas teniendo en cuenta este potencial, y lo hacemos adecuadamente, tendremos en nuestras manos un instrumento de aprendizaje privilegiado. Debe existir una coherencia interna en el diseño de actividades, medios y materiales, secuencias y tiempos, así como en criterios y procedimientos de evaluación que constituyen un aspecto fundamental.

La formación del profesorado novel es un aspecto que está suscitando cada vez más interés en las universidades españolas. Sin embargo, nuestra experiencia de más de veinte años como docentes universitarios nos lleva a afirmar que, al menos en nuestro ámbito más cercano, las áreas de ciencias experimentales y de la salud, todavía se está en una etapa preliminar. Se plantea a continuación la experiencia seguida en el seno de un equipo docente, constituido ante la necesidad de avanzar en estos aspectos, y se recogen los principales resultados alcanzados durante su trayectoria.

\section{DESCRIPCIÓN DE LA EXPERIENCIA}

En este apartado se describe la experiencia desarrollada durante tres cursos consecutivos. Seguidamente se presentan los objetivos y por último se detalla la metodología utilizada para la formación en evaluación formativa y compartida.

Primer curso. Los antecedentes del actual equipo docente se encuentran en nuestra participación en la $2^{\mathrm{a}}$ edición del Curso de Formación de Profesores Asesores (2009), organizado por el Vicerrectorado para la Garantía de la Calidad de la Universidad de Granada. Como resultado de dicho curso se elaboró un programa de mentorización que se desarrolló en la fase de tutorización de la $2^{\mathrm{a}}$ edición del Curso de Formación Inicial para la Docencia Universitaria, organizado por dicho Vicerrectorado.

Las fases del programa de mentorización se presentan en la figura 1, integrando en el ciclo la actuación del equipo docente y su actividad de consolidación en el proceso de formación.

De las acciones realizadas en dicho programa cabe destacar los ciclos de mejora desarrollados en primer lugar entre mentores y, a continuación, entre parejas mentor-novel. Un ciclo de mejora es una estrategia cooperativa de desarrollo profesional mediante la cual los participantes utilizan un "apoyo colegiado para adquirir un mayor control personal sobre el conocimiento obtenido acerca de su propia enseñanza y aprendizaje" y debe servir para impulsarlos a implicarse activamente en la mejora de sus actuaciones docentes (Sánchez y Mayor, 2006). 


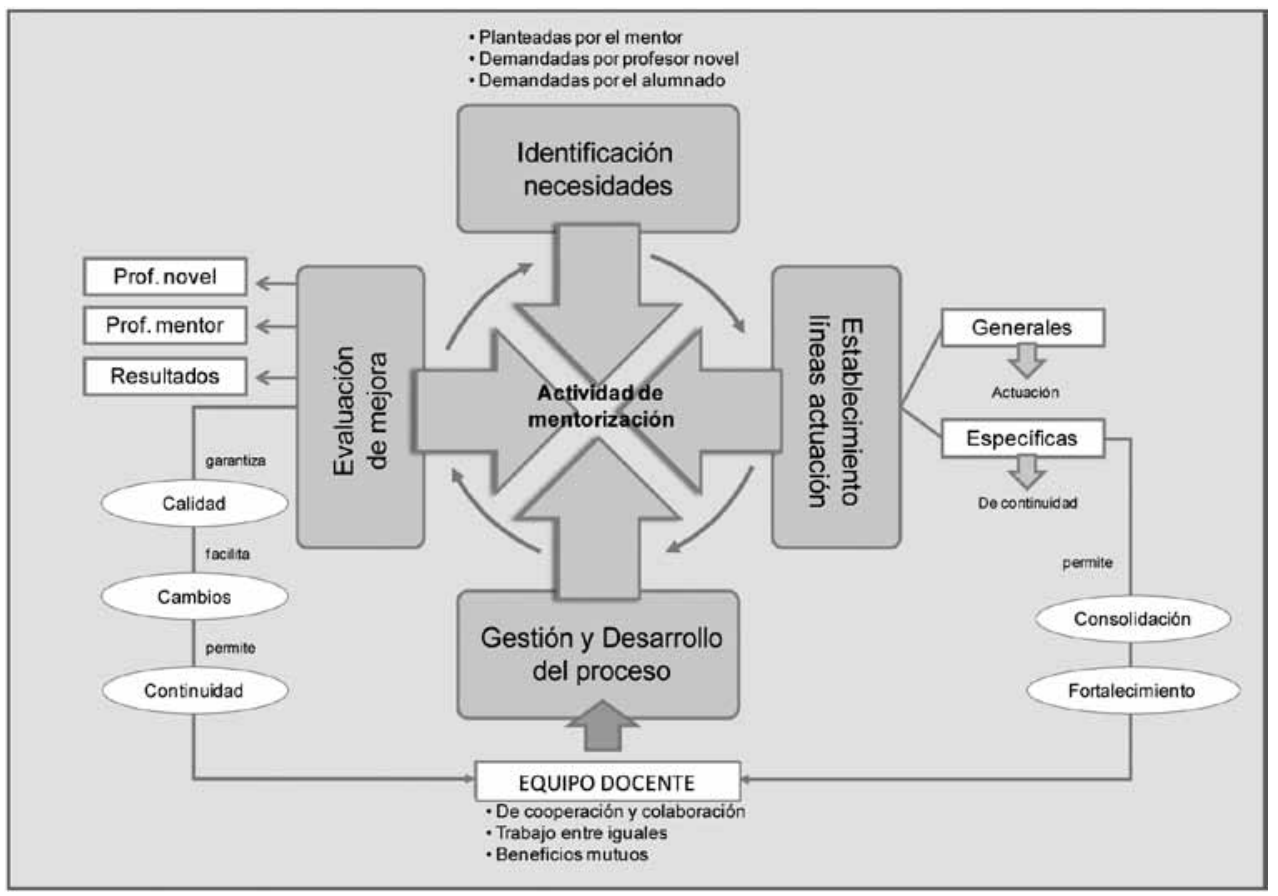

Figura 1. Fases del programa de mentorización (Curso 2009/2010).

El objetivo de un ciclo de mejora es la planificación, observación y análisis de las actuaciones docentes llevadas a cabo por el profesorado y consta de cuatro fases:

a. Entrevista de planificación. Se decide sobre qué aspecto o aspectos concretos, que puedan ser observables directamente, se va a trabajar, partiendo siempre de los intereses del novel.

b. Observación. Se realiza según lo planificado en la etapa anterior. La observación no es un acto informal e imprevisto sino que se trata de una actividad sistemática y organizada (Sánchez y Mayor, 2006). En nuestro caso se utilizaron grabaciones en video ya que proporcionan un material muy valioso que se puede revisar tantas veces como sea necesario.

c. Análisis. Mentor y novel analizan independientemente los datos recogidos en la observación, centrándose en el aspecto objeto de estudio. Para ello se emplean los test de observación directa que permiten analizar el trabajo del profesor en el aula, su relación con los estudiantes, la utilización de materiales y métodos didácticos, el desarrollo de contenidos y su conocimiento y dominio del tema (Sánchez, 1993; Cerezo, Bosque, del Olmo y Plaza, 2010).

d. Entrevista de análisis. Es el momento de poner en común y reflexionar 
conjuntamente a partir del análisis previo. El mentor en esta etapa es crucial ya que puede contribuir en gran medida al éxito o al fracaso del ciclo de mejora. El profesor novel es un colega que puede tener puntos de vista diferentes en cuanto a la forma de plantearse su propia docencia. La labor del mentor no es decirle cómo tiene que actuar sino ayudarle a reflexionar sobre sus debilidades, plantearle interrogantes donde no los haya y orientarle para que encuentre sus propias soluciones acordes con su personalidad y sus expectativas. "Reflexionar sobre qué se hace y sobre por qué se hace lo que se hace" (Ángel y Amor, 2007 y Mayor, 2009). Es decir, no se trata de actuar como un consejero experto sino de ayudar al principiante en su proceso de identificación como profesor universitario.

Como paso previo a la labor con los noveles decidimos realizar ciclos de mejora entre mentores para poner a punto la estrategia. Además, se nos presentaba una magnífica herramienta para analizar nuestra propia práctica docente con otros colegas. Esta experiencia fue innovadora y muy enriquecedora para todos $\mathrm{y}$, entre otros aspectos positivos, sirvió para cohesionar a los miembros del grupo. Queremos destacar que ésta fue la primera vez en nuestra vida profesional (20 años en promedio) que fuimos observados, analizados y dejamos cuestionar nuestra práctica docente "real" por otros colegas.

La evaluación del programa de mentorización (Bosque, Cerezo, del Olmo y Plaza, 2010a) permitió detectar las fortalezas y debilidades de todo el proceso, destacando el alto grado de satisfacción de los participantes en la experiencia.

Segundo curso. A partir de la labor desarrollada en el programa de mentorización se decide formalizar el equipo docente participando en la Convocatoria de Apoyo a la Formación del Profesorado Principiante y Mejora de la Docencia 2010 .

El equipo docente está constituido por cinco profesores experimentados pertenecientes a las áreas de Farmacia y Tecnología Farmacéutica, Química Analítica y Química Física y trece principiantes vinculados a las tres áreas mencionadas, además de Genética e Ingeniería Química. La docencia se imparte también en varias titulaciones: Bellas Artes, Biología, Ciencias Ambientales, Farmacia, Ingeniería Química y Química.

Entre los profesores principiantes cabe destacar la diversidad en su experiencia docente: ocho de ellos se encuentran en su primer año con dedicación docente como becarios predoctorales; el resto lleva tres, cuatro y hasta cinco años como becarios y actualmente tienen distintos tipos de contratos. Además, hay que señalar que cinco de ellos son responsables de asignaturas, mientras que el resto participa en la docencia de prácticas de laboratorio.

El programa de formación se centra en profundizar y experimentar en aspectos concretos que son fundamentales en la acción docente. También se ha procurado que fuera flexible y estuviera abierto a posibles cambios para atender 
las necesidades demandadas por el profesorado novel y las posibles carencias detectadas durante el proceso. Las acciones desarrolladas han sido las siguientes:

I. La evaluación como instrumento de aprendizaje. El trabajo llevado a cabo en esta línea del programa se detalla en el apartado "La evaluación como instrumento de aprendizaje en la formación del profesorado universitario".

II. La comunicación en la acción docente. La comunicación tiene una importancia fundamental, tanto en el aula como en la relación más personalizada con el alumnado fuera de la misma, en tutorías por ejemplo (Bimbela y Navarro, 2005). Sin embargo, se presupone que el profesorado universitario posee las habilidades comunicativas mínimas necesarias para que este aspecto primordial se desarrolle con éxito. Creemos que una formación específica, y eminentemente práctica, en habilidades comunicativas debe abordarse en la formación del profesorado novel y que, sin duda, también así se favorece la reflexión sobre la propia práctica diaria del profesorado experimentado. Para desarrollar esta acción se han realizado distintos talleres, en los que se ha trabajado, mediante ejercicios de dinámica de grupos, aspectos relevantes en el proceso comunicativo cómo comunicación no verbal, retroalimentación y asertividad, entre otros.

III. Utilización de las TICs en docencia universitaria, fue una línea propuesta por el profesorado novel.

IV. Ciclos de mejora. Se han utilizando como instrumento para analizar y mejorar la actividad docente del profesorado participante en el programa. Dichos ciclos se han desarrollado como se ha indicado anteriormente.

Tercer curso. Las acciones que se están desarrollando en la continuación del programa (convocatoria 2011) son:

I. La evaluación como instrumento de aprendizaje. Se continua la labor ya iniciada, fruto de la cual varios de los componentes del equipo se han integrado en la Red Nacional de Evaluación Formativa y Compartida en Docencia Universitaria (López, Castejón, Sicilia-Camacho, NavarroAdelantado y Webb, 2011).

II. Habilidades docentes e inteligencia emocional. Diversos estudios publicados recientemente (Palomera, Fernández-Berrocal y Brackett, 2008; Rietveldt, Fernández y Luquez, 2009) avalan la conveniencia de abordar las competencias socioemocionales en la formación inicial y permanente del profesorado universitario. Estas competencias se enmarcan en el modelo de inteligencia emocional difundido por Goleman (1997), que permite conocer, valorar, controlar y mejorar tanto nuestras propias emociones y sentimientos como los del alumnado para conseguir una enseñanza y un aprendizaje más eficaces. El equipo ya se ha iniciado en 
este sentido trabajando específicamente las habilidades comunicativas. Considerando el interés mostrado por el profesorado principiante y los buenos resultados de la valoración de dicha acción, se sigue avanzando en esta dirección abordando las competencias socioemocionales.

III. Ciclos de mejora. Se están enfocando especialmente a aquellas competencias y habilidades a las que se refieren las nuevas acciones planteadas.

\section{Objetivos}

Los objetivos generales del programa son los propuestos en la Convocatoria de Apoyo a la Formación del Profesorado Principiante y Mejora de la Docencia 2010 (Vicerrectorado para la Garantía de la Calidad, Universidad de Granada, 2010) . Entre ellos, ofrecer al profesorado principiante una formación específica que le ayude a optimizar su actividad docente e impulsar la creación y consolidación de un grupo docente estable, comprometido en la formación inicial del profesorado novel y en la mejora permanente de la docencia.

Por otra parte, los objetivos específicos están directamente relacionados con las acciones concretas del programa Formación compartida de la actividad docente del profesorado universitario ante el EEES (Bosque, Cerezo, del Olmo y Plaza, 2010b). Entre ellos, desarrollar y/o potenciar una actitud autorreflexiva y crítica sobre la propia práctica docente, mejorar la capacidad del profesorado experimentado para liderar un grupo en formación y reflexionar sobre la necesidad de hacer de la evaluación un instrumento de aprendizaje.

La evaluación como instrumento de aprendizaje en la formación del profesorado universitario

Durante el segundo curso se han realizado una serie de actividades dirigidas a la formación, reflexión y puesta en práctica de estrategias de evaluación formativa y compartida, según la secuencia que se recoge a continuación:

1. Seminario-taller sobre fundamento teórico, técnicas e instrumentos. Impartido por un miembro de la Red de Evaluación Formativa y Compartida en Docencia Universitaria (López, 2009).

2. Diseño de estrategias de evaluación formativa y compartida. Se plantearon un total de siete propuestas de forma individual o en equipos de dos o más profesores. Dos de ellas estaban dirigidas de forma exclusiva a su desarrollo durante las sesiones de trabajo experimental y guiadas por profesorado novel en su primer año de docencia, otra abarcaba una asignatura en su conjunto y el resto se centraban en el trabajo en el aula.

3. Seminario de puesta en común. Discusión y valoración en grupo de las estrategias diseñadas.

4. Desarrollo de las actividades planificadas. Tuvo lugar durante el segundo semestre, salvo en las asignaturas anuales, pertenecientes a los antiguos 
planes de estudio, gestionadas por profesorado experimentado y que iniciaron su experiencia con el curso académico.

5. Seminario de seguimiento. Se realizó en el mes de abril, coincidiendo aproximadamente con la mitad del semestre.

6. Seminario final de valoración. Desarrollado una vez finalizado el periodo lectivo.

7. Realización de un portafolio. En él se recogía tanto la evolución del proceso como las reflexiones de los docentes.

Durante el tercer curso se continúa trabajando en esta línea de formación, centrándonos en aspectos concretos como la retroalimentación durante el proceso, la autoevaluación o la evaluación entre compañeros. También queremos conocer y experimentar metodologías activas que faciliten la evaluación formativa y la motivación de los alumnos.

\section{EVALUACIÓN DE LA EXPERIENCIA DE INNOVACIÓN}

\section{Herramientas de evaluación}

En cualquier programa de formación se debe establecer un método que permita extraer conclusiones sobre la bondad de las actividades realizadas así como sobre el grado de satisfacción alcanzado por los participantes. Con ello se pretende evaluar las mejoras alcanzadas y por otra, recibir una retroalimentación que permita realizar cambios con objeto de mejorar la continuación del programa.

La evaluación incluyó la cumplimentación de una encuesta tras cada una de las actividades y otra global de todo el proceso. Asimismo, se mantuvo una reunión con todos los participantes para realizar la valoración final del trabajo realizado en el equipo.

1. Evaluación de las actividades realizadas. Se pretendía obtener información sobre la satisfacción e importancia que cada participante concede a aspectos como: Metodología utilizada, cualificación de los docentes, nivel de reflexión y mejora de la interpretación del tema tratado, grado de aplicabilidad de los contenidos y grado de satisfacción por la formación práctica adquirida.

2. Evaluación global del programa de formación. Los elementos del programa a valorar fueron: Contenido, metodología, organización, docentes, ambiente y relaciones, actividad formativa y grado de satisfacción.

3. Reunión de valoración final. Se discutieron las fortalezas y debilidades del proceso global así como las propuestas de mejora para la continuación del programa. 


\section{Principales resultados alcanzados}

En primer lugar se presentan los correspondientes al programa y seguidamente los relativos a la acción: La evaluación como instrumento de aprendizaje en la formación del profesorado universitario.

Programa de Formación. Son destacables los buenos resultados obtenidos en las encuestas por actividades y en la evaluación global del programa. En todos los casos las puntuaciones estaban por encima de 4 sobre un máximo de 5 , tanto en la satisfacción alcanzada como en la importancia atribuida, poniéndose de manifiesto un alto grado de cumplimiento de las expectativas iniciales. Asimismo, todos los participantes manifestaron su interés en continuar profundizando en estas líneas de formación.

En la reunión final se discutieron las fortalezas, debilidades y propuestas de mejora.

Como fortalezas cabe señalar la reflexión sobre la actividad docente desarrollada a lo largo de todo el proceso, la labor realizada por los profesores experimentados y su grado de compromiso y dedicación, el buen ambiente alcanzado y las excelentes relaciones interpersonales así como la gran motivación existente en todos los participantes. El alto nivel de los expertos externos y la metodología seguida en los talleres se han subrayado de manera explícita ya que han permitido adquirir nuevos conocimientos y habilidades de aplicación inmediata al proceso enseñanza-aprendizaje. Finalmente, la coincidencia del programa con el inicio de los nuevos grados, elaborados según las directrices del EEES, ha facilitando la adaptación a las nuevas exigencias formativas.

Como debilidades se puede destacar que el programa era demasiado ambicioso, lo que ha requerido una dedicación excesiva; además, después de las distintas actividades se realizaron un número reducido de reuniones de reflexión conjunta y extracción de conclusiones y finalmente, el uso poco acertado del portafolio como sistema de evaluación.

Como propuestas de mejora planteadas podemos señalar la necesidad de un mayor número de reuniones para la reflexión conjunta y el seguimiento de las actividades propuestas, la reducción del intervalo de tiempo entre actividades relacionadas y la utilización de un sistema de evaluación más adecuado a las características del equipo.

La evaluación como instrumento de aprendizaje en la formación del profesorado universitario. La actividad realizada dentro del equipo docente para la formación, reflexión y puesta en práctica de estrategias de evaluación formativa y compartida ha tenido un apreciable éxito. Es destacable que la mayoría de los profesores participantes han manifestado su interés en esta metodología, utilizándola durante el curso 2010/2011 en las asignaturas que se imparten dentro de diversas Licenciaturas y/o Grados. Los cinco profesores experimentados y 
diez de los trece profesores principiantes participaron en el seminario inicial y se implicaron en el diseño y desarrollo de las experiencias.

En el marco de esta experiencia se ha elaborado diverso material curricular (matrices de valoración, fichas de observación de competencias y distintos cuestionarios de evaluación, entre otros) para su aplicación en el proceso de evaluación formativa y compartida de asignaturas de ciencias experimentales, donde su uso es aún muy restringido y por tanto también son escasos los materiales didácticos existentes.

En la Tabla 1 se recogen algunos de los resultados obtenidos en la encuesta realizada sobre esta actividad. En concreto, los relativos a la percepción de los participantes sobre la utilidad de esta metodología docente.

Tabla 1. Grado de Satisfacción y Opinión de Los Participantes En La Actividad De Evaluación Formativa y Compartida (Valorados En Una Escala Del 1 Al 5, Donde 5 Corresponde a La Mayor Satisfacción o Importancia)

\begin{tabular}{|llc|}
\hline \multicolumn{1}{|c}{ Aspecto Evaluado } & $\begin{array}{c}\text { Satisfacción } \\
\text { Valor medio } \\
\text { (Desviación } \\
\text { estándar) }\end{array}$ & $\begin{array}{c}\text { Importancia } \\
\text { Valor medio } \\
\text { (Desviación } \\
\text { estándar) }\end{array}$ \\
$\begin{array}{l}\text { Cumplimiento de las expectativas generadas. } \\
\text { Nivel de nuevos conocimientos adquiridos. }\end{array}$ & $4,2(1,0)$ & $4,6(0,5)$ \\
$\begin{array}{l}\text { Grado de aplicabilidad de los contenidos } \\
\text { desarrollados. }\end{array}$ & $3,9(1,2)$ & $4,6(0,5)$ \\
$\begin{array}{l}\text { Grado de satisfacción por la formación práctica } \\
\text { adquirida. }\end{array}$ & $4,3(1,0)$ & $4,4(0,7)$ \\
$\begin{array}{l}\text { ¿Te interesa seguir profundizando sobre el } \\
\text { tema? }\end{array}$ & \multicolumn{2}{c}{ SI $(86 \%)$} \\
\hline
\end{tabular}

A partir del análisis de estos resultados y de lo expresado por el profesorado participante en la reunión de valoración se puede destacar:

- Los profesores, tanto principiantes como experimentados, han recibido formación e información sobre evaluación formativa y compartida y han reflexionado sobre su potencial para mejorar el aprendizaje y valorar competencias que no son observables con otros métodos. El profesorado ha valorado positivamente la formación recibida y considera de gran interés su utilización. Por ello, han diseñado y experimentado con estas metodologías en las asignaturas impartidas.

- Por otra parte, los estudiantes, en general, han mostrado bastante interés y aceptación por las acciones docentes realizadas. Se ha observado que el número de alumnos que deciden abandonar la asignatura (no presen- 
tados), es superior en el caso de los grupos evaluados con esta metodología. Asimismo, se alcanza una mejora en las calificaciones finales y se percibe un incremento en la motivación y el interés de los alumnos. Adicionalmente, allí donde se han implantando sistemas de evaluación compartida se ha observado una mejora en la capacidad de análisis crítico y autocrítico de los alumnos, que ha reforzado su aprendizaje.

\section{CONCLUSIONES}

En este artículo se describe la trayectoria de un equipo docente constituido por profesorado novel y experimentado perteneciente a áreas de ciencias experimentales y de la salud. La experiencia se limita a un periodo de dos cursos académicos y continúa en la actualidad. Para los participantes en este proyecto, el trabajo colaborativo en el equipo está suponiendo el inicio de una nueva manera, al menos en nuestro ámbito más cercano, de plantearnos nuestra formación, tanto inicial como permanente, como docentes universitarios.

Uno de los logros más inmediatos es el asesoramiento que se está proporcionando al profesorado principiante que contribuye a reducir la inseguridad e incertidumbres propias del inicio de la carrera docente y fomenta una cultura del desarrollo profesional basada en la reflexión sobre la propia práctica.

Por otro lado, se está motivando a todos los participantes, noveles y experimentados, a explorar otras metodologías que mejoren el proceso enseñanzaaprendizaje. De esta forma el equipo funciona como espacio de reflexión conjunta dónde se favorece el espíritu crítico, el intercambio y confrontación de experiencias, la observación de "buenas prácticas", el fomento de la innovación, etc. En este sentido cabe destacar las innovaciones realizadas en evaluación formativa y compartida que están provocando un replanteamiento de la programación de diversas asignaturas.

En definitiva, la experiencia desarrollada nos permite concluir que estos equipos docentes formados por profesorado novel y experimentado pueden ser un instrumento muy útil para la formación inicial y permanente de los docentes universitarios. Seguramente esto sea debido a que se implica activamente al propio profesorado en el diseño de dicha formación y lo hace protagonista y corresponsable de la misma, junto con la institución universitaria.

\section{AGRADECIMIENTOS}

Al Vicerrectorado para la Garantía de la Calidad de la Universidad de Granada por la financiación del programa (ref. 2010-08).

\section{REFERENCIAS}

Ángel, P., y Amor, P. (2007). Guía práctica del coaching. Barcelona: Paidós. Bimbela Pedrosa, J. L., y Navarro Matillas, B. (2005). Cuidando al formador. 
Habilidades emocionales y de comunicación. Granada: Escuela Andaluza de Salud Pública.

Bosque Sendra, J. M., Cerezo González, P., del Olmo Iruela, M., y Plaza del Pino, I. M. (2010a). Mentorización de profesorado novel. Creación de una red docente entre las facultades de ciencias y farmacia. En M. J. León Guerrero, y M. C. López López (coord.), Actas Primeras Jornadas Andaluzas de Formación Inicial del Profesorado Universitario (pp. 181-190). Granada: Universidad de Granada.

Bosque Sendra, J. M., Cerezo González, P., del Olmo Iruela, M., y Plaza del Pino, I. M. (2010b). Formación compartida de la actividad docente del profesorado universitario ante el EEES. Recuperado el 17 de diciembre de 2011, de http:/calidad.ugr.es/pages/secretariados/form_apoyo_calidad/programa_ iniciacion_docencia/equipos-docentes-2010/formacian-compartida-de-laactividad-docente-del-profesorado-universitario-ante-el-eees

Brown, S., y Glasner, A. (2003). Evaluar en la universidad. Problemas y nuevos enfoques. Madrid: Narcea.

Cerezo González, M. P., Bosque Sendra, J. M., del Olmo Iruela, M., y Plaza del Pino, I. M. (2010). Propuesta de un instrumento para la observación de la competencia docente del profesorado universitario. En Actas III Congreso Internacional de Educación Superior en Ciencias Farmacéuticas (pp. 157). Granada: Sider S. C.

Dochy, F., Segers, M., y Dierick, S. (2002). Nuevas vías de aprendizaje y enseñanza y sus consecuencias: una nueva era de evaluación. Boletín de la Red Estatal de Docencia Universitaria 2 (1), 11-28. Recuperado el 5 de junio de 2011, de http://www.redu.um.es/publicaciones/vol2_n2.htm\#dochy

Escudero Muñoz, J. M. (2009). El proceso de iniciación a la práctica profesional y el asesoramiento entre iguales. (Curso de Formación para profesores asesores, $2^{\mathrm{a}}$ ed.). Vicerrectorado para la Garantía de la Calidad, Universidad de Granada.

Goleman, D. (1997). La inteligencia emocional. Barcelona: Kairós.

López Pastor, V. (coord.) (2009). Evaluación Formativa y Compartida en Educación Superior. Madrid: Narcea.

López Pastor, V., Castejón, J., Sicilia-Camacho, A., Navarro-Adelantado, V., y Webb, G. (2011). The process of creating a cross-university network for formative and shared assessment in higher education in Spain and its potential applications. Innovations in Education and Teaching International, 48, 7990.

Madrid Izquierdo, J. M. (2005). La formación y la evaluación docente del profesorado universitario ante el Espacio Europeo de Educación Superior. Educatio, 23, 49-68.

Mayor Ruiz, C. (2009). Nuevos retos para una universidad en proceso de cambio: ¿Pueden ser los profesores (principiantes) los protagonistas? Profesorado. Revista de currículum y formación del profesorado, 13 (1), 61-77.

Mayor Ruiz, C., y Sánchez Moreno, M. (1999). Los equipos docentes: una con- 
tribución formativa a la calidad del profesorado universitario. XXI. Revista de Educación, 1, 157-176. Recuperado el 18 de diciembre de 2011, de http:// www.uhu.es/publicaciones/ojs/index.php/xxi/article/view/555

Palomera, R., Fernández-Berrocal, P., y Brackett, M. A. (2008). La inteligencia emocional como una competencia básica en la formación inicial de los docentes: algunas evidencias. Revista Electrónica de Investigación Psicoeducativa, 6 (2), 437-454.

Rietveldt F., Fernández O., y Luquez P. (2009). Inteligencia emocional y competencias del docente universitario. Revista Ciencias de la Educación, 19 (3), 126-143.

Sánchez Moreno, M. (1993). La supervisión clínica como estrategia de formación de profesores principiantes y mentores. Tesis no publicada, Facultad de Ciencias de la Educación, Universidad de Sevilla, Sevilla, España.

Sánchez Moreno, M. (2008). Asesoramiento en la universidad. Poniendo a trabajar a la experiencia. Profesorado. Revista de currículum y formación del profesorado, 12 (1), 1-19.

Sánchez Moreno, M., y Mayor Ruiz, C. (2006). Los jóvenes profesores universitarios y su formación pedagógica. Claves y controversias. Revista de Educación, 339, 923-946.

Sanmartí, N. (2007). 10 Ideas Clave. Evaluar para Aprender. Barcelona: Grao.

Vicerrectorado para la Garantía de la Calidad, Universidad de Granada (2010). Convocatoria de Apoyo a la Formación del Profesorado Principiante y Mejora de la Docencia 2010. Recuperado el 17 de diciembre de 2011, de http:// calidad.ugr.es/pages/secretariados/form_apoyo_calidad/apoyo_formacion principiante/2010/convocatoria 2010 
